\title{
Electron Microscopy of Nuclear Membranes during Somatic Nuclear Separation in Neurospora crassa
}

\author{
By T. EGASHIRA, J. TOKUNAGA AND MICHIKO TOKUNAGA \\ Laboratory of Electronmicroscopy, Kyushu Dental College, Kitakyushu, Japan
}

(Accepted for publication I7 January 1972)

\begin{abstract}
Moore (1963, I964) described karyochorisis in Cordyceps in which the invagination of the inner nuclear membrane divided the nucleoplasm into two or more subunits and subsequent invagination of outer nuclear membrane separated the nucleus into daughter nuclei. In Catenaria the telophase stage of mitosis is marked by the rounding up of the daughter nuclei with the exclusion from the nucleus of a central, spindle-shaped structure which is then broken down (Ichida, I968). A similar configuration has been shown in meiosis of Physarum (Aldrich, I967).

This paper deals with nuclear division in germinating conidia of Neurospora crassa.
\end{abstract}

\section{METHODS}

Conidia formed by cultures of Neurospora crassa (FGSC 821) grown on minimal agar medium (Beadle \& Tatum, I945) for 6 days at $25^{\circ} \mathrm{C}$, were harvested with distilled water and separated from hyphae by filtration through glass wool.

Samples were incubated at $30^{\circ} \mathrm{C}$ in minimal liquid medium and centrifuged. Germinating conidia were fixed in $2 \%(\mathrm{w} / \mathrm{v})$ aqueous $\mathrm{KMnO}_{4}$ or in $6 \%(\mathrm{v} / \mathrm{v})$ aqueous glutaraldehyde followed by postfixation in $2 \%(\mathrm{w} / \mathrm{v})$ aqueous $\mathrm{KMnO}_{4}$.

The samples were dehydrated in an ethanol series, rinsed in propylene oxide, and then embedded in a mixture of Epon 812 and Epok 533. Sections were cut with glass knives on an LKB ultramicrotome, stained with uranyl acetate and lead citrate (Reynolds, 1963), and examined with a JEM-7 electron microscope.

\section{RESULTS}

Fig. I ( $a$ ) shows a nucleus with an invagination of the inner nuclear membrane. The outer membrane was clearly continuous across the point of invagination. No nucleus showed simultaneous membranous invaginations on both sides of the dividing nucleus, indicating that the invagination proceeded from one side of the nuclear surface to the other and not centripetally.

Ultimately, an invagination of the inner nuclear membrane seemed to fuse with the nuclear membrane at the opposite side of the nuclear surface, resulting in a partitioned nucleus as seen in Fig. $\mathrm{I}(b)$.

At the next stage of division, the nucleus showed a partition wall consisting of four parallel membranes. Fig. I $(c)$ probably shows two daughter nuclei immediately after nuclear division, each nucleus being completely enclosed by a double-membraned envelope.

Fig. I $(d)$ shows a nucleus that had apparently divided into four parts by the invagination 

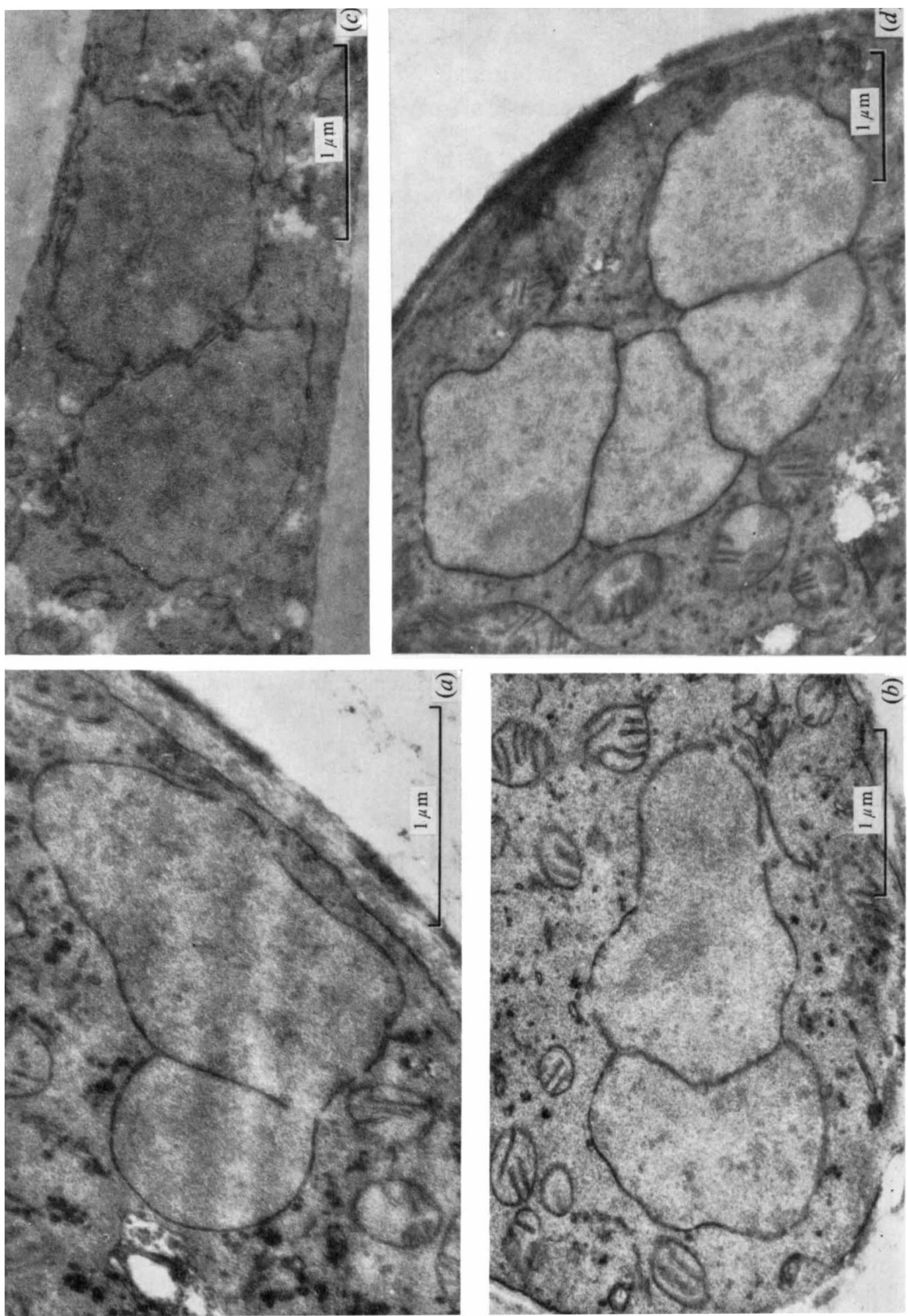
of the inner nuclear membrane at three points. Each partition probably consisted of two membranes. This would eliminate the possibility that some of the nuclei associated together to produce the present nuclear configuration.

\section{DISCUSSION}

The process of nuclear separation in Neurospora crassa which closely resembles that in Cordyceps (Moore, I964), including the occurrence of the multipartitioned nucleus as shown in Fig. $\mathrm{I}(d)$. The additional membranes may have been formed by further invagination of the outer nuclear membrane, as described in Cordyceps (Moore, 1964), or by accumulation of membranous component substances on to either the outside or the inside of the invaginated inner membrane.

Tandler, Erlandson, Smith \& Wynder (1969) have reported that dividing mitochondria in the liver of mice given an injection of riboflavin are characterized by a membranous partition separating the inner compartment into two parts. This is accomplished either by fusion of existing cristae, or by elongation of a single crista, followed by the extension of the outer membrane of the mitochondrial envelope between the component membranes of the septum, resulting in a separation of the two mitochondrial moieties, perhaps due to a repulsive force between the outer membranes. The same may be a possible mechanism of nuclear separation in Neurospora.

The type of somatic nuclear separation in Neurospora is certainly different from yeast cells (Hawker, 1965) and Catenaria (Ichida, 1968). Occurrence of multiseparated nucleus may indicate that the daughter nuclei initiated the next division before their separation was completed.

The only report on nuclear division of Neurospora studied by electron microscopy has been published by Namboodiri \& Lowry (1967). They showed two slender nuclei lying parallel to each other in hyphae of mutant 'clock', which they interpreted as two daughter nuclei immediately after telophase. Although they showed disintegration of nuclear envelope during the early stage of division, this was not observed in the present study. No configuration indicating the invagination of nuclear membrane was shown by Namboodiri \& Lowry (1967).

We express our gratitude to Drs E. B. Wagenaar \& K. Nakamura, Department of Biological Sciences, University of Lethbridge, for their comments and advice.

Fig. I (a) Invagination of the inner nuclear membrane. The outer membrane is clearly continuous across the point of invagination.

Fig. I (b). A nucleus partitioned by the invagination of the inner membrane.

Fig. I (c). Accomplishment of nuclear separation. Note the daughter nuclei surrounded by intact double membranes.

Fig. I $(d)$. Multipartitioned nucleus resulting from several simultaneous invaginations of the inner nuclear membrane. 


\section{REFERENCES}

ALDRICH, H. C. (1967). The ultrastructure of meiosis in three species of Physarum. Mycologia 59, 127-148.

BeAdLE, G. W. \& TATUM, E. L. (1945). Neurospora. II. Methods of producing and detecting mutations concerned with nutritional requirements. American Journal of Botany 32, 678-685.

HAWKer, L. E. (1965). Fine structure of fungi as revealed by electron microscopy. Biological Reviews 40, 52-92.

ICHIDA, A. A. (1968). Ultrastructure of mitosis in the aquatic fungus Catenaria anguillulae. Mycologia 6o, I4I-I 55 .

MOORE, R. T. (1963). Ultrastructure of somatic nuclear division in Cordyceps militaris. American Journal of Botany 50, 626.

MoORE, R. T. (1964). Fine structure of Mycota. I2. Karyochorisis - somatic nuclear division - in Cordyceps militaris. Zeitschrift für Zellforschung und mikroskopische Anatomie 63, 921-937.

Namboodiri, A. N. \& Lowry, R. J. (1967). Vegetative nuclear division in Neurospora. American Journal of Botany 54, 735-748.

REYNOLDS, E. S. (1963). The use of lead citrate at high $\mathrm{pH}$ as an electronopaque stain in electron microscopy. Journal of Cell Biology 17, 208-212.

TANDler, B., Erlandson, R. A., Smith, A. L. \& Wynder, E. L. (I969). Riboflavin and mouse hepatic cell structure and function. II. Division of mitochondria during recovery from simple deficiency. Journal of Cell Biology 4r, 477-493. 